\title{
A Structured Approach to Solve the Inverse Eigenvalue Problem for a Beam with Added Mass
}

\author{
Farhad Mir Hosseini and Natalie Baddour \\ Department of Mechanical Engineering, University of Ottawa, 161 Louis Pasteur, Ottawa, ON, Canada K1N 6N5 \\ Correspondence should be addressed to Natalie Baddour; nbaddour@uottawa.ca
}

Received 31 October 2013; Revised 17 January 2014; Accepted 24 January 2014; Published 12 March 2014

Academic Editor: Herb Kunze

Copyright (C) 2014 F. Mir Hosseini and N. Baddour. This is an open access article distributed under the Creative Commons Attribution License, which permits unrestricted use, distribution, and reproduction in any medium, provided the original work is properly cited.

\begin{abstract}
The problem of determining the eigenvalues of a vibrational system having multiple lumped attachments has been investigated extensively. However, most of the research conducted in this field focuses on determining the natural frequencies of the combined system assuming that the characteristics of the combined vibrational system are known (forward problem). A problem of great interest from the point of view of engineering design is the ability to impose certain frequencies on the vibrational system or to avoid certain frequencies by modifying the characteristics of the vibrational system (inverse problem). In this paper, a method to impose two natural frequencies on a dynamical system consisting of an Euler-Bernoulli beam and carrying a single mass attachment is evaluated.
\end{abstract}

\section{Introduction}

The problem of determining the eigenvalues (natural frequencies) of a combined dynamical system has been the subject of extensive research in the past. One of the combined dynamical systems whose vibrational analysis is of great interest is a beam to which several lumped elements are attached. These lumped elements can take different forms such as point or rotary masses, translational as well as torsional springs, and translational as well as torsional dampers.

The majority of the research performed in this area involves the development and evaluation of methods to determine the natural frequencies of the combined system assuming that the characteristics of the combined system are known (forward problem). Kukla and Posiadala in [1] and Nicholson and Bergman in [2] employed a Green's function method to derive the exact solution for the frequency of a combined dynamical system. Kukla and Posiadala considered the problem of determining the frequencies of beams with elastically mounted masses and obtained the exact solution for the frequency of the transversal vibrations of the beam in closed form while Nicholson and Bergman derived the exact solution for two types of linear undamped systems, one with one rigid body degree of freedom (a spring-mass system hung from the beam) and the other with no rigid body degree of freedom (a grounded spring attached to a lumped mass). In [3], Dowell generalized the results of the Rayleigh's method for the calculation of the frequency of combined mechanical systems. Unlike Rayleigh's method, this approach states that the natural frequency of the combined systems increases in every condition. The same authors in [4] investigated the application of Lagrange multipliers to the analysis of the free vibrations of different structures including beams. Low et al. [5-8] considered the problem of determining the frequencies of a combined dynamical system in several papers. In [5], they performed the frequency analysis of a beam with attached concentrated masses and the effects of the positions and values of the masses on the natural frequencies of the combined system. The exact solution to the eigenvalue problem of the frequency of a beam with concentrated masses was established. In [6], they took on the task of deriving a transcendental equation for the frequency calculation of a beam with single mass attachments and compared this with Rayleigh's method. In [7], they compared two methods of deriving the frequency equation of a beam with lumped mass attachments, namely, a determinant method, using the Laplace transform. They maintained the same approach in [8] where they compared the eigenanalysis (exact) and 
Rayleigh's methods to solve for the frequencies of a beam with multiple mass attachments. Cha et al. in [9] proposed a method to calculate the natural frequencies (eigenvalues) of a beam with multiple miscellaneous lumped attachments by reducing the order of the matrices whose determinants are to be solved from $N$ (number of assumed modes) to $S$ (number of attachments). In another paper, Cha and Yoder [10] considered the free and forced vibration of beams carrying lumped elements in the form of point masses and translational as well as torsional springs and dampers using Sherman-Morrison as well as Sherman-Morrison-Woodbury formulas.

Some researchers considered the sensitivity of the natural frequencies of the combined system to the parameters of the attached elements. In [11], Wang considered the effect and sensitivity of positioning lumped (concentrated) masses on an Euler-Bernoulli beam on the beam's natural frequencies. Using finite element analysis, a closed-form expression for the frequency sensitivity with respect to the mass location was obtained. Pritchard et al. [12] also considered sensitivity and optimization studies with regard to the node locations of a beam to which lumped masses were attached. Analytical and Finite Element Method (FEM) results were compared with corresponding results using the finite difference method.

As far as the inverse problem is concerned, Cha and Pierre [13] used the assumed-modes method to propose a forward solution for determining the frequencies of a linear elastic structure (beam) to which a system of masses and springs was attached at a particular point. This approach then provided a method of solving the inverse problem of imposing nodes at a specific location. For this to happen, the frequency of the combined system must be equal to the frequency of the grounded mass-spring system, that is, the natural frequency of the isolated system of the mass and the linear spring $(\omega=\sqrt{k / m})$. The same authors in [14] analyzed the inverse problem of imposing nodes along a beam using a combination of elastically mounted masses. Using a system of masses and springs, an analytical method was developed to make it possible to impose nodes in a desired location along a beam with arbitrary boundary conditions.

As we have outlined above, most of the research performed in this area concerns the forward problem of determining the eigenvalues (natural frequencies) of a combined dynamical system assuming that all of its physical characteristics are known. In a few cases where the inverse problem is considered, the authors were concerned with imposing nodes on specified locations along the beam. However, another important inverse problem is the ability to impose certain natural frequencies on the system or to avoid others by modifying the parameters of the lumped attachments. This problem has widespread engineering applications, from the construction of musical instruments to the design of aircraft wings.

In this paper, the authors propose and evaluate a method to impose two desired natural frequencies on a dynamical system of an Euler-Bernoulli beam to which a single lumped mass is attached by determining the magnitude and mounting position of the mass.

\section{Theory}

2.1. The Method of Assumed Modes. Many approaches utilized to solve continuous problems in engineering involve the discretization of the continuous system into elements for which analytical or numerical solutions can be found. One of the most widely used methods is Finite Element Analysis (FEA) which involves the discretization of the continuous system into a number of small, discrete elements and the application of compatibility conditions at the interface of the adjacent elements as well as the application of boundary conditions. The greater the number of elements utilized, the more accurate the results obtained.

For the special case of vibrational analysis, there exists another commonly used discretization approach, called the assumed modes method. The logic behind this method is the principle of superposition of different vibrational modes that the system may undergo. As with the case of FEA, the greater the number of modes utilized, the more accurate the results obtained. However, in contrast to FEA, assumed modes are a superposition of global elements, with each element often defined over the entire domain of the problem. Usually, the vibrational modes of a related but simpler problem are superimposed to find approximate solutions to a more complicated problem. A good introduction to the assumed modes can be found in [15].

Both of these methods, when applied to a continuous, conservative vibrational system, will result in two matrices, namely, mass and stiffness matrices. The dimensions of these matrices are determined by the degree of discretization selected for the problem. Here lies the main advantage of the assumed modes method over finite element analysis. It has been shown in [9] that the same level of accuracy can be reached by the assumed modes method using smaller degrees of discretization than with FEA. This implies mass and stiffness matrices that are smaller and can be handled more easily as far as computational issues are concerned.

For this paper, the assumed modes method was chosen to derive the equations of motion for the case of an EulerBernoulli beam to which a number of discrete elements are attached. The discretization process starts with modelling the transverse vibrations of an Euler-Bernoulli beam as a finite series whose elements are the product of an eigenfunction and a generalized coordinate so that the transverse vibrations of the beam can be written as

$$
w(x, t)=\sum_{j=1}^{N} \phi_{j}(x) \eta_{j}(t) .
$$

Here, $w(x, t)$ is the transverse displacement of the beam, $\phi_{j}(x)$ is a space-dependent eigenfunction, $\eta_{j}(t)$ is the generalized coordinate, and $N$ is the number of assumed modes chosen for the problem. It is important to note that $\phi_{j}(x)$ varies with the choice of the beam and any $\phi_{j}(x)$ should be chosen to satisfy the required geometric boundary conditions of the selected beam.

As can be seen in (1), the eigenfunctions are functions of position, $x$, and the generalized coordinates are only a function of time, $t$, which demonstrates the application of separation of variables in this method. 


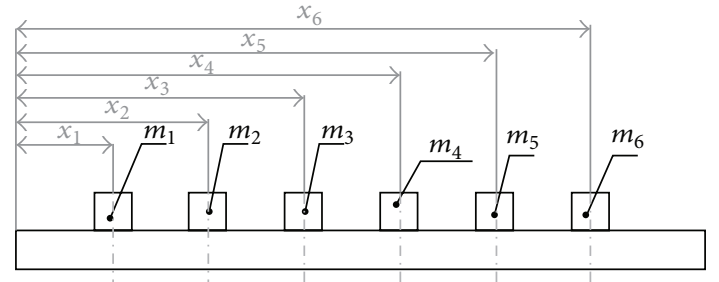

FIGURE 1: Beam with multiple lumped mass attachments.

2.2. Derivation of Equations of Motion. In order to derive the equations of motion for the one dimensional Euler-Bernoulli beam with multiple lumped point-mass attachments as can be seen in Figure 1, expressions for kinetic and potential energies must first be found. The kinetic energy of the beam is given by

$$
T=\frac{1}{2} \sum_{j=1}^{N} M_{j} \dot{\eta}_{j}^{2}(t)+\frac{1}{2} m_{1} \dot{w}^{2}\left(x_{1}, t\right)+\cdots+\frac{1}{2} m_{s} \dot{w}^{2}\left(x_{s}, t\right),
$$

where $M_{j}$ are generalized masses of the bare beam (no attachments), an over dot indicates derivatives with respect to time, and $m_{1} \cdots m_{s}$ are $s$ lumped point masses positioned at $x_{1} \cdots x_{s}$, respectively.

Using the same procedure, the equation for the potential energy can be written as

$$
V=\frac{1}{2} \sum_{j=1}^{N} K_{j} \eta_{j}^{2}(t)
$$

where $K_{j}$ are the generalized stiffnesses of the bare beam.

Substituting (1) into (2), the following equation for kinetic energy is obtained:

$$
\begin{aligned}
T= & \frac{1}{2} \sum_{j=1}^{N} M_{j} \dot{\eta}_{j}^{2}(t) \\
& +\frac{1}{2} m_{1}\left[\sum_{j=1}^{N} \phi_{j}\left(x_{1}\right) \dot{\eta}_{j}(t)\right]^{2} \\
& +\cdots+\frac{1}{2} m_{s}\left[\sum_{j=1}^{N} \phi_{j}\left(x_{s}\right) \dot{\eta}_{j}(t)\right]^{2} .
\end{aligned}
$$

Equation (3) for the potential energy remains the same as no elastic element is added to the beam.

Having found the expressions for kinetic and potential energies in terms of $\phi$ and $\eta$, these are then substituted into the Lagrange's equations to yield the equations of motion. Lagrange's equations are given by

$$
\frac{d}{d t}\left(\frac{\partial T}{\partial \dot{\eta}_{i}}\right)-\frac{\partial T}{\partial \eta_{i}}+\frac{\partial V}{\partial \eta_{i}}=0 \quad i=1,2, \ldots, N
$$

where $N$ corresponds to the number of generalized coordinates and hence the number of differential equations.
Substituting (4) and (3) into (5) and converting the system of equations into a matrix representation, the matrix equation of motion will be given by

$$
\mathbf{M} \ddot{\ddot{\eta}}+\mathbf{K} \underline{\eta}=\underline{0},
$$

where $\mathbf{M}$ and $\mathbf{K}$ are the system mass and stiffness matrices, respectively, and $\mathbf{M}$ is given by

$$
\mathbf{M}=\mathbf{M}^{d}+m_{1} \cdot \underline{\phi}_{1} \cdot \underline{\phi}_{1}^{T}+\cdots+m_{s} \cdot \underline{\phi}_{s} \cdot \underline{\phi}_{s}^{T} .
$$

In (7), $\underline{\phi}_{1} \cdots \underline{\phi}_{s}$ are $N$-dimensional column vectors of the $N$ eigenfunctions evaluated at the attachment points $x_{1} \cdots x_{s}$, so that, for example,

$$
\underline{\phi}_{1}=\left[\begin{array}{c}
\phi_{1}\left(x_{1}\right) \\
\vdots \\
\phi_{N}\left(x_{1}\right)
\end{array}\right] .
$$

$\mathbf{M}^{d}$ is a diagonal matrix whose diagonal components are the generalized masses $M_{i}$ and $m_{1} \cdots m_{s}$ are the masses of the lumped attachments.

As far as the stiffness matrix is concerned, since elastic elements are not being added to the beam, it remains a diagonal matrix whose elements are the generalized stiffnesses of the beam. Hence, the stiffness matrix is given by

$$
\mathbf{K}=\mathbf{K}^{d} .
$$

2.3. Choice of Eigenfunctions. As can be seen in (7) and (8), eigenfunction constitutes a major component of the mass matrix of the combined system. It is solely a function of position, $x$, and can take many different forms depending on the beam being considered. The most basic requirement of any eigenfunction simulating the vibrational behaviour of a beam is its ability to accommodate and satisfy the beam boundary conditions. In this paper, the eigenfunction for an Euler-Bernoulli beam with simply-supported boundary conditions is given by [9]

$$
\phi_{i}(x)=\sqrt{\frac{2}{\rho L}} \sin \left(\frac{i \pi x}{L}\right),
$$

where $\rho$ is the mass per unit length of the beam and $L$ represents the length of the beam.

On the other hand, for the case of an Euler-Bernoulli beam with fixed-free boundary conditions, the eigenfunction is given by [9]

$$
\begin{aligned}
\phi_{i}(x)=\frac{1}{\sqrt{\rho L}}( & \cos \beta_{i} x-\cosh \beta_{i} x \\
& \left.+\frac{\sin \beta_{i} L-\sinh \beta_{i} L}{\cos \beta_{i} L+\cosh \beta_{i} L}\left(\sin \beta_{i} x-\sinh \beta_{i} x\right)\right),
\end{aligned}
$$

where $\beta_{i} L$ 's are calculated using the following transcendental equation:

$$
\cos \left(\beta_{i} L\right) \cosh \left(\beta_{i} L\right)=-1
$$


2.4. Frequencies and Mode Shapes. In order to solve (6), a system of $N$ second-order differential equations, the vector of generalized coordinates $\underline{\eta}$ is written as

$$
\underline{\eta}=\vec{\eta} e^{i \omega t}
$$

Here, $\omega$ is the frequency of vibration of the system. Moreover, the inclusion of the complex number " $i$ " is justified given the fact that the system is conservative and it is expected that the vibrations are purely oscillatory.

Substituting (13) into (6) and taking derivatives yield

$$
\left(-\omega^{2} \mathbf{M}+\mathbf{K}\right) \vec{\eta}=\underline{0} .
$$

In order for (14) to have a nontrivial solution, the following equation must hold:

$$
\operatorname{det}\left(-\omega^{2} \mathbf{M}+\mathbf{K}\right)=0 .
$$

Substituting $\lambda=\omega^{2}$ in (15), an equivalent expression is obtained:

$$
\operatorname{det}(\mathbf{K}-\lambda \mathbf{M})=0
$$

Equation (16) can be solved for either the squared frequencies of the system $\lambda$ (forward problem) or the coefficients of $\mathbf{K}$ and $\mathbf{M}$ matrices (inverse problem). The code for the forward modelling problem was verified against the results in [16], which in turn were verified against Finite Element Method solutions. In this paper, the case of inverse problem is investigated for the case of an Euler-Bernoulli beam with a single mass attachment.

2.5. Inverse Problem. The problem of imposing two frequencies on a dynamical system consisting of a beam with a single attached lumped mass is considered here which leads to the following system of equations:

$$
\begin{aligned}
& \operatorname{det}\left(\mathbf{K}-\lambda_{a} \mathbf{M}\right)=0 \\
& \operatorname{det}\left(\mathbf{K}-\lambda_{b} \mathbf{M}\right)=0,
\end{aligned}
$$

where $\lambda_{a}$ and $\lambda_{b}$ are the desired natural frequencies squared to be imposed on the system (design variables). Due to the fact that no stiffness element is added to the beam, $\mathbf{K}$ remains intact and can be determined using (9) while the mass matrix of the combined system $\mathbf{M}$ is given by

$$
\mathbf{M}=\mathbf{M}^{d}+m_{1} \cdot \underline{\phi}_{1} \cdot \underline{\phi}_{1}^{T},
$$

where $m_{1}$ is the magnitude of the lumped mass and $\underline{\phi}_{1}$ is a function of the mass position, $x_{1}$, and is defined by (8). $m_{1}$ and $x_{1}$ are the unknown variables of the inverse problem. Substituting (18) into (17), a system of two equations and two unknowns is obtained whose solution is presented in the next section for the case of two commonly used boundary conditions: simply-supported as well as fixed-free.

Due to the fact that the two equations are derived using (17) which includes the determinant, this method is referred to as the Determinant Method and was first introduced in [17]. In using the determinant method, each desired natural frequency is substituted into a determinant equation of the form of (17). Therefore, each desired natural frequency produces a single equation. Therefore, $n$ desired natural frequencies require $n$ "design degrees of freedom" or parameters to be controlled, such as added masses or springs, or their location on the beam. The value of the added mass (or spring) is considered as one design degree of freedom and its unknown location is another. The form of the equations produced via imposing a desired frequency on the determinant (such as (17)) depends on the choice of eigenfunctions in the assumed modes method and also how the unknown desired parameter (e.g., position of the mass) is included in those eigenfunctions. In this manuscript, we have chosen the traditional trigonometric functions as the eigenfunctions since they are the most well-known choices for the EulerBernoulli beam. Other choices of eigenfunctions may lead to better computational results with this method.

\section{Results}

The following assumptions are considered in defining the inverse problem.

(i) Only the case of adding a single mass is considered.

(ii) The known (input) variables are two desired natural frequencies that must be imposed on the system.

(iii) The unknown variables to be found are the mass and its position along the beam.

(iv) The acceptable mass range is a fraction of the mass of the beam; that is, $m=c \rho L$ for $0<c<1$.

(v) The inverse problem is solved for both the cases of simply-supported and fixed-free (cantilever) boundary conditions.

(vi) The degree of discretization using assumed modes is $N=10$ for simply-supported beam and $N=4$ for fixed-free (cantilever) beam.

(vii) The acceptable position range is a fraction of the length of the beam $L$; that is, $p L$, where $0 \leq p \leq 1$.

Using Maple V14 (Maplesoft), the inverse problem was coded for the two cases of simply-supported as well as fixed-free boundary conditions.

3.1. Simply-Supported Beam. The major steps in solving, as well as coding, the inverse eigenvalue problems are outlined here. First, two natural frequencies are chosen as desired input frequencies $\left(\lambda_{a}, \lambda_{b}\right)$. These are the frequencies we seek to impose on the beam with its mass attachment. To insure a solvable problem, we choose known values from previously solved forward problem. The generalized mass and stiffness 
TABLE 1: Inverse problem for 2nd and 3rd frequencies for a simply-supported beam.

\begin{tabular}{|c|c|c|}
\hline Input given to inverse determinant method & Solution via inverse determinant method & $\begin{array}{c}\text { Full span of frequency squared spectrum } \\
\text { obtained via the solution of forward problem }\end{array}$ \\
\hline \multirow{20}{*}{$\begin{array}{l}\lambda_{2}=1210.7430, \lambda_{3}=7703.7093 \\
\text { (obtained with } m=0.2 \rho L \\
l=0.3 L \text { in the forward code) }\end{array}$} & \multirow{12}{*}{$\begin{array}{c}\qquad \begin{array}{c}m=0.2 \rho L \\
l=0.3 L\end{array} \\
\text { (this result was obtained via } \\
\text { both DirectSearch and fsolve methods) }\end{array}$} & 76.8682 \\
\hline & & 1210.7430 \\
\hline & & 7703.7093 \\
\hline & & 22705.2481 \\
\hline & & 49733.6143 \\
\hline & & $1.19385 \times 10^{5}$ \\
\hline & & $2.29571 \times 10^{5}$ \\
\hline & & $3.45310 \times 10^{5}$ \\
\hline & & $5.95631 \times 10^{5}$ \\
\hline & & $9.74091 \times 10^{5}$ \\
\hline & & 59.0673 \\
\hline & & 1210.7430 \\
\hline & \multirow{8}{*}{$\begin{array}{c}\qquad m=0.3895 \rho L \\
l=0.3587 L \\
\text { (this result was obtained via } \\
\text { both DirectSearch and fsolve packages) }\end{array}$} & 7703.7093 \\
\hline & & 18122.3022 \\
\hline & & 55694.1292 \\
\hline & & $1.18846 \times 10^{5}$ \\
\hline & & $1.91164 \times 10^{5}$ \\
\hline & & $3.88304 \times 10^{5}$ \\
\hline & & $5.89699 \times 10^{5}$ \\
\hline & & $8.69163 \times 10^{5}$ \\
\hline
\end{tabular}

matrices must be formed $\left(\mathbf{M}^{d}, \mathbf{K}^{d}\right)$ whose diagonal elements are given by

$$
\begin{gathered}
M_{i}=1, \\
K_{i}=\frac{(i \pi)^{4} E I}{\left(\rho L^{4}\right)} .
\end{gathered}
$$

The eigenfunction vector, $\phi$, must also be built using the simply-supported beam eigenfunction equation (10). The stiffness matrix is unaffected since no stiffness elements is added. The mass matrix of the combined system is affected by the presence of lumped masses. Hence, the matrices are given by

$$
\begin{gathered}
\mathbf{K}_{t}=\mathbf{K}^{d}, \\
\mathbf{M}_{t}=\mathbf{M}^{d}+c \rho L \underline{\phi}_{p L} \underline{\phi}_{p L}^{T},
\end{gathered}
$$

where $c$ is the mass coefficient and vector $\underline{\phi}_{p L}$ is defined as

$$
\underline{\phi}_{p L}=\left[\begin{array}{c}
\phi_{1}(p L) \\
\vdots \\
\phi_{N}(p L)
\end{array}\right] .
$$

Equation (20), along with the two desired values of $\lambda$ are substituted into (17). This gives two equations in two unknowns which will be solved for the two unknowns, $c$ and $p$, using fsolve as well as the DirectSearch packages in Maple. Fsolve is Maple's built-in equation-solving package. The details of the DirectSearch package can be found in [18]. The results obtained for $c$ and $p$ include the anticipated results (already known from the forward problem since we chose the desired $\lambda$ from a known forward problem) plus additional results for $c$ and $p$. To check whether the order of the frequencies in the frequency spectrum will be conserved or if the results returned by the inverse problem achieve the desired system frequencies, the parameters $c$ and $p$ must be substituted into the forward code in order to obtain the entire spectrum of system frequencies. Due to the symmetry of the simply-supported boundary condition about the beam midpoint, only half of the beam is considered and the results can be extended to the other half. These results are tabulated in the left hand columns of Tables 1, 2, and 3.

The squared frequencies in the left hand side columns of these tables are chosen from the previously solved forward problem and their subscripts indicate their order in the hierarchy of the frequency spectrum. The middle columns of these tables contain the values of the masses as well as their positions on the beam obtained after substituting the squared frequencies of the first columns into the inverse problem code. Finally, the right hand side columns are the full span of frequency spectrum obtained after substituting the masses as 
TABLE 2: Inverse problem for 2nd and 4th frequencies for a simply-supported beam.

\begin{tabular}{|c|c|c|}
\hline Input given to inverse determinant method & Solution via inverse determinant method & $\begin{array}{l}\text { Full span of frequency squared spectrum } \\
\text { obtained via the solution of forward problem }\end{array}$ \\
\hline \multirow{30}{*}{$\begin{array}{l}\lambda_{2}=1411.9003, \lambda_{4}=20420.5236 \\
\text { (obtained with } m=0.2 \rho L \\
l=0.4 L \text { in the forward code) }\end{array}$} & \multirow{10}{*}{$\begin{array}{c}m=0.1516 \rho L \\
l=0.0972 L \\
\text { (this result was obtained via } \\
\text { DirectSearch and fsolve packages) }\end{array}$} & 94.7851 \\
\hline & & 1411.9003 \\
\hline & & 6626.2231 \\
\hline & & 20420.5236 \\
\hline & & 51205.1150 \\
\hline & & $1.11210 \times 10^{5}$ \\
\hline & & $2.15698 \times 10^{5}$ \\
\hline & & $3.82314 \times 10^{5}$ \\
\hline & & $6.29638 \times 10^{5}$ \\
\hline & & $9.73288 \times 10^{5}$ \\
\hline & \multirow{11}{*}{$\begin{array}{l}\qquad m=0.4695 \rho L \\
\quad l=0.4268 L \\
\text { (this result was obtained via } \\
\text { DirectSearch package only) }\end{array}$} & 51.2467 \\
\hline & & 1411.9003 \\
\hline & & 6082.1204 \\
\hline & & 20420.5235 \\
\hline & & 57662.4808 \\
\hline & & $1.00203 \times 10^{5}$ \\
\hline & & $2.33797 \times 10^{5}$ \\
\hline & & $3.33408 \times 10^{5}$ \\
\hline & & $6.17507 \times 10^{5}$ \\
\hline & & $9.04206 \times 10^{5}$ \\
\hline & & 71.3527 \\
\hline & \multirow{9}{*}{$\begin{array}{c}\qquad m=0.2 \rho L \\
l=0.4 L \\
\text { (this result was obtained } \\
\text { via DirectSearch package only) }\end{array}$} & 1411.9003 \\
\hline & & 7164.6511 \\
\hline & & 20420.5236 \\
\hline & & 60880.6819 \\
\hline & & $1.05946 \times 10^{5}$ \\
\hline & & $2.21752 \times 10^{5}$ \\
\hline & & $3.77388 \times 10^{5}$ \\
\hline & & $5.73630 \times 10^{5}$ \\
\hline & & $9.74091 \times 10^{5}$ \\
\hline
\end{tabular}

well as their positions of the middle column into the forward problem. The desired squared frequencies are in bold face in the right hand column vectors to make it easier for the reader to compare them with the desired frequencies of the left hand side column.

By considering the left hand column of Tables 1 and 2 , the following observations can be made regarding the solution to the inverse frequency (eigenvalue) problem. First, the order of the two desired system frequencies was conserved. For instance, in Table 2, the two desired input frequencies remained as the 2 nd and 4 th system frequencies when the full span of the frequency spectrum was found for all three possible solutions. Second, due to the symmetry of the boundary conditions of the simply-supported beam, it is evident that for each mass obtained, there must be two corresponding positions that are symmetrical with respect to the middle of the beam. Finally, a good equation solver is a requirement for this method to work properly and in this case, the use of an alternative equation solver (DirectSearch) yielded additional parameters $c$ and $p$ that were not returned by Maple's built-in equation solver. For example, In Table 2, the third result is obtained using the DirectSearch package only.

We consider another example with the simply-supported beam where the chosen frequencies are the 2nd and 8th natural frequencies, in order to verify that the method works when the chosen natural frequencies are not chosen to be close together, namely, when $\lambda i$ and $\lambda j$ are specified and 
TABLE 3: Inverse problem for 2nd and 8th frequencies for a simply-supported beam.

\begin{tabular}{|c|c|c|}
\hline Input given to the inverse problem & Solution via inverse determinant method & $\begin{array}{c}\text { Full span of frequency squared spectrum } \\
\text { obtained via the solution of forward problem }\end{array}$ \\
\hline \multirow{40}{*}{$\begin{array}{l}\lambda_{2}=1411.900275, \lambda_{8}=3.773881670 \times 10^{5} \\
\text { (obtained with } m=0.2 \rho L \\
l=0.4 L \text { in the forward code) }\end{array}$} & \multirow{14}{*}{$\begin{aligned} m & =0.2 \rho L \\
l & =0.6 L\end{aligned}$} & 71.3527 \\
\hline & & 1411.9003 \\
\hline & & 7164.65111 \\
\hline & & 20420.52363 \\
\hline & & 60880.6819 \\
\hline & & $1.05946 \times 10^{5}$ \\
\hline & & $2.21752 \times 10^{5}$ \\
\hline & & $3.77388 \times 10^{5}$ \\
\hline & & $5.73630 \times 10^{5}$ \\
\hline & & {$\left[9.74091 \times 10^{5}\right]$} \\
\hline & & 86.6441 \\
\hline & & 1411.9003 \\
\hline & & 7887.5523 \\
\hline & & 22430.0485 \\
\hline & \multirow{7}{*}{$\begin{array}{c}m=0.0807 \rho L \\
l=0.6615 L\end{array}$} & 56495.1732 \\
\hline & & $1.26101 \times 10^{5}$ \\
\hline & & $2.13015 \times 10^{5}$ \\
\hline & & $3.77388 \times 10^{5}$ \\
\hline & & $6.37810 \times 10^{5}$ \\
\hline & & {$\left[9.05601 \times 10^{5}\right]$} \\
\hline & & [ 94.2653 \\
\hline & \multirow{9}{*}{$\begin{aligned} m & =0.0752 \rho L \\
l & =0.8447 L\end{aligned}$} & 1411.9003 \\
\hline & & 6953.8085 \\
\hline & & 22707.0792 \\
\hline & & 58428.8453 \\
\hline & & $1.25669 \times 10^{5}$ \\
\hline & & $2.31928 \times 10^{5}$ \\
\hline & & $3.77388 \times 10^{5}$ \\
\hline & & $5.86872 \times 10^{5}$ \\
\hline & & {$\left[9.10470 \times 10^{5}\right]$} \\
\hline & \multirow{10}{*}{$\begin{aligned} m & =0.0585 \rho L \\
l & =0.7133 L\end{aligned}$} & 90.83562 \\
\hline & & 1411.9003 \\
\hline & & 7750.6323 \\
\hline & & 24432.2348 \\
\hline & & 55584.5685 \\
\hline & & $1.20289 \times 10^{5}$ \\
\hline & & $2.33870 \times 10^{5}$ \\
\hline & & $3.77388 \times 10^{5}$ \\
\hline & & $5.98869 \times 10^{5}$ \\
\hline & & $9.64184 \times 10^{5}$ \\
\hline
\end{tabular}


TABLE 3: Continued.

\begin{tabular}{|c|c|c|}
\hline Input given to the inverse problem & Solution via inverse determinant method & $\begin{array}{l}\text { Full span of frequency squared spectrum } \\
\text { obtained via the solution of forward problem }\end{array}$ \\
\hline & \multirow{10}{*}{$\begin{aligned} m & =0.0573 \rho L \\
l & =0.7944 L\end{aligned}$} & 93.4974 \\
\hline & & 1411.9003 \\
\hline & & 7262.2914 \\
\hline & & 24320.8984 \\
\hline & & 60836.8705 \\
\hline & & $1.20776 \times 10^{5}$ \\
\hline & & $2.15464 \times 10^{5}$ \\
\hline & & $3.77388 \times 10^{5}$ \\
\hline & & $6.30690 \times 10^{5}$ \\
\hline & & $9.72121 \times 10^{5}$ \\
\hline
\end{tabular}

when $|i-j|$ is larger. These results are shown in Table 3. As can be seen from the table, the method worked without difficulty in this case, although it was observed that a larger number of possible solutions were obtained than in the prior cases shown in Tables 1 and 2. From the three tables for the simply-supported beam, we note that the larger the separation between $\lambda \mathbf{i}$ and $\lambda \mathbf{j}$ was (in their chosen order in the spectrum), the greater the number of possible solutions to the inverse problem was obtained.

3.2. Cantilever (Fixed-Free) Beam. The steps to follow in order to code the inverse problem for the cantilever beam follow the same steps as for the simply-supported beam. However, due to additional complexity of the eigenfunction of a fixed-free (cantilever) beam (11), additional steps are required at the outset of the code. In particular, the transcendental equation of (12) must be solved and the generalized stiffness elements must be replaced by

$$
K_{i}=\frac{\left(\beta_{i} L\right)^{4} E I}{\left(\rho L^{4}\right)} .
$$

Additionally, due to the complexities of the eigenfunction and thus resulting determinant, the highest degree of discretization that Maple V14 could handle for the inverse problem was found to be $N=4$. For discretization degrees greater than $N=4$, each equation obtained from evaluating the two determinants of (17) contained more than one million terms. This was a result of the choice of eigenfunction, with the chosen eigenfunction for the cantilever beam containing more terms than the corresponding one for the simplesupported beam. This highlights how the choice of assumed modes for the forward problem affects the complexity of the inverse problem and in fact its solvability. In this paper, we proceed with a parallel choice of eigenfunctions as assumed modes for both simply-supported and cantilever beams to highlight how a specific type of eigenfunction that performs well in the inverse problem for one type of boundary condition may not be as useful when the boundary conditions of the problem are changed. Choosing assumed modes to ensure or enhance solvability of a particular inverse problem will be considered in future work.

Moreover, since the investigation of the inverse problem for the simply-supported beam suggests that DirectSearch package yields more results compared to the built-in fsolve package, DirectSearch was used to solve (17). The rest of the procedure follows the same steps as for the case of a simplysupported beam.

A pair of frequencies was chosen from the already solved forward problem and then substituted into the inverse code as the desired system frequencies. In the same manner as for the simply-supported beam, the resulting equations of motion were then solved to yield the masses and their corresponding positions from (17). The results of the inverse code were then substituted into the forward problem to determine the full span of frequency spectrum as well as the order of the desired pair of frequencies in the hierarchy of the frequency spectrum. The results of these simulations are shown in Tables 4 and 5. Moreover, in Tables 4 and 5, the left hand side columns are the pair of squared frequencies chosen from the solution of forward problem with a degree of discretization $N=10$; the indices indicate their orders in the hierarchy of the frequency spectrum. The middle columns contain the values of the masses and their corresponding locations along the beam after substituting the pair of squared frequencies of the left hand side column into the inverse code whose degree of discretization is $N=4$. This degree of discretization $(N=$ 4) was chosen to ensure solvability of the inverse problem as Maple had difficulties in solving the inverse problem for the cantilever beam with higher orders of discretization. Finally, the right hand side columns contain the full span of the frequency spectrum after substituting the values of the masses and their corresponding locations along the beam of the middle columns back into the forward problem code whose degree of discretization was chosen as $N=10$.

By considering Tables 4 and 5, the following observations can be made regarding the inverse eigenvalue (frequency) problem of a fixed-free beam. First, despite the fact that the degrees of discretization for forward and inverse problems are very different, $N=10$ and $N=4$, respectively, 
TABLE 4: Inverse problem solution for 2nd and 3rd imposed frequency for a cantilever beam.

\begin{tabular}{lll} 
Input given to the inverse problem $\quad$ Solution via inverse determinant method $\quad \begin{array}{c}\text { Full span of frequency squared spectrum } \\
\text { obtained via the solution of forward problem }\end{array}$ \\
\hline
\end{tabular}

12.2709

435.8829

3147.4731

13829.1182

$m=0.1010 \rho L$

39445.9834

$l=0.2989 L$

78488.2022

$1.61140 \times 10^{5}$

$3.08184 \times 10^{5}$

$\lambda_{2}=435.9606, \lambda_{3}=3153.6181$

$4.68642 \times 10^{5}$

(obtained with $m=0.1 \rho L$

$7.26151 \times 10^{5}$

$l=0.3 L$ in the forward code)

10.6171

435.8075

3234.8623

14601.0784

34510.6316

79566.7957

$1.73775 \times 10^{5}$

$2.71381 \times 10^{5}$

$4.75801 \times 10^{5}$

$7.89162 \times 10^{5}$ the results of the full frequency spectrum obtained after substituting the mass and its corresponding position into the forward problem still show good approximation with respect to the original frequencies. This is evident by comparing the input frequencies on the left hand side columns with the bold numbers in the vector of squared frequencies in the right hand side columns of Tables 4 and 5 . The results of our proposed approach could be used as starting values for an optimization algorithm [19-21] if more precise results are required, where careful selection of the optimization approach will depend on the intended outcome of the design/optimization process. Second, as with the case of the simply-supported beam, the order of the frequencies in the full span of frequency spectrum remains the same for all mass and position solutions. Third, the effect of the degree of discretization is most noticeable in the higher order frequencies. In other words, the higher the order of frequency, the higher the divergence from the exact solution. This implies that for situations where the lower natural frequencies are of concern, lower degrees of discretization suffice for the engineering design purposes which imply lower order matrices, in addition to lower order polynomials and thus less computation (see Tables 4 and 5). For example, in the design of musical instruments, there is common consensus that the lower modes of vibration are of greater importance [22-24].

\section{Inverse Simply Supported Beam Problem with Two Masses}

To investigate the possibility of using the determinant method with more than one mass, we considered the problem of imposing four frequencies on a dynamical system consisting of a beam with two attached lumped masses. Following our previous development, this leads to the following system of equations:

$$
\begin{aligned}
& \operatorname{det}\left(\mathbf{K}-\lambda_{a} \mathbf{M}\right)=0, \\
& \operatorname{det}\left(\mathbf{K}-\lambda_{b} \mathbf{M}\right)=0, \\
& \operatorname{det}\left(\mathbf{K}-\lambda_{c} \mathbf{M}\right)=0, \\
& \operatorname{det}\left(\mathbf{K}-\lambda_{d} \mathbf{M}\right)=0,
\end{aligned}
$$

where $\left\{\lambda_{a}, \lambda_{b}, \lambda_{c}, \lambda_{d}\right\}$ are the desired natural frequencies squared to be imposed on the system (design variables). Due to the fact that no stiffness element is added to the beam, $\mathbf{K}$ remains intact and can be determined using (3) while the mass matrix of the combined system $\mathbf{M}$ is now given by

$$
\mathbf{M}=\mathbf{M}^{d}+m_{1} \cdot \underline{\phi}_{1} \cdot \underline{\phi}_{1}^{T}+m_{2} \cdot \underline{\phi}_{2} \cdot \underline{\phi}_{2}^{T},
$$


TABLE 5: Inverse problem for 1st and 2nd imposed natural frequencies for a cantilever beam.

\begin{tabular}{|c|c|c|}
\hline Input given to the inverse problem & Solution via inverse determinant method & $\begin{array}{l}\text { Full span of frequency squared spectrum } \\
\text { obtained via solution of forward problem }\end{array}$ \\
\hline \multirow{20}{*}{$\begin{array}{l}\lambda_{1}=12.3554, \lambda_{2}=476.9708 \\
\text { (obtained with } m=0.5 \rho L \\
l=0.1 L \text { in the forward code) }\end{array}$} & \multirow{10}{*}{$\begin{aligned} m & =0.5 \rho L \\
l & =0.1 L\end{aligned}$} & 12.3553 \\
\hline & & 476.8083 \\
\hline & & 3358.6521 \\
\hline & & 10287.9858 \\
\hline & & 25821.5612 \\
\hline & & 61911.2963 \\
\hline & & $1.32415 \times 10^{5}$ \\
\hline & & $2.53985 \times 10^{5}$ \\
\hline & & $4.46546 \times 10^{5}$ \\
\hline & & $7.32837 \times 10^{5}$ \\
\hline & \multirow{10}{*}{$\begin{array}{c}m=0.6047 \rho L \\
l=0.0954 L\end{array}$} & 12.3554 \\
\hline & & 476.7893 \\
\hline & & 3344.4265 \\
\hline & & 10049.3118 \\
\hline & & 25096.4956 \\
\hline & & 60477.9117 \\
\hline & & $1.29731 \times 10^{5}$ \\
\hline & & $2.49237 \times 10^{5}$ \\
\hline & & $4.38699 \times 10^{5}$ \\
\hline & & $7.20790 \times 10^{5}$ \\
\hline
\end{tabular}

where $m_{1}$ and $m_{2}$ are the magnitudes of the lumped masses, $\phi_{1}$ is a function of the first mass position $x_{1}, \phi_{2}$ is a function of the second mass position $x_{2}$ and both $\underline{\phi}_{1}$ and $\underline{\phi}_{2}$ are defined by equation (8). The variables $m_{1}, m_{2}, x_{1}$, and $x_{2}$ are the unknown variables of the inverse problem. Substituting (24) into (23), a system of four equations and four unknowns is obtained whose solution is presented in Table 6 for the case of a simply-supported beam. In these simulations, $N=$ 8 was used and both fsolve and DirectSearch were used to solve the inverse problem. The parameters used to obtain the desired frequencies (found via the forward problem) are: $m_{1}=0.2 \rho L, x_{1}=0.2 L$ and $m_{2}=0.8 \rho L, x_{2}=0.7 L$. Maple's built-in fsolve package returned only one solution $m_{1}=0.1878 \rho L, l_{1}=0.3164 L$ and $m_{2}=0.5629 \rho L, l_{2}=$ $0.8001 L$. Although these parameters are not the same as the original parameters used to obtain the desired frequencies, they are in fact a solution to the problem since they return a system with exactly the desired frequencies. The package DirectSearch returned 96 possible solutions, of which 57 were physically plausible. Due to the high number of possible solutions returned by DirectSearch, we only illustrate three possible solutions obtained by DirectSearch in Table 6, in addition to the lone solution returned by fsolve. In all cases, any solutions obtained by this determinant method approach to the inverse problem must be substituted back into the forward problem in order to verify that the results are correct or are within the required tolerance of the design problem at hand.

\section{Conclusion}

A method to impose two natural frequencies on a dynamical system consisting of a beam to which a single lumped mass is attached is evaluated. In this method, the known (design) variables are the two natural frequencies and the unknown variables are the magnitude of the attached mass as well as its position along the beam. The proposed method is easy to code and can accommodate any kind of eigenfunctions. The results are obtained for two commonly used boundary conditions, namely, simply-supported and cantilever. It is shown that the expected values of the added mass and its position are recovered from the inverse problem, in addition to additional unexpected values. This demonstrates that even for this simple problem, a unique solution does not exist. The nonuniqueness of the solution can be considered as a benefit from the point of view of design possibilities. An investigation of the inverse problem also reveals that the order of the frequencies in the hierarchy of the whole frequency spectrum is conserved. Although ideally the degree of discretization should be the same for both forward and inverse problem, it is observed that lower degrees of discretization for the inverse problem still yield acceptable results from the point 
TABLE 6: Inverse problem for 2nd, 4th, 6th, and 8th frequencies for a simply-supported beam with two mass attachments.

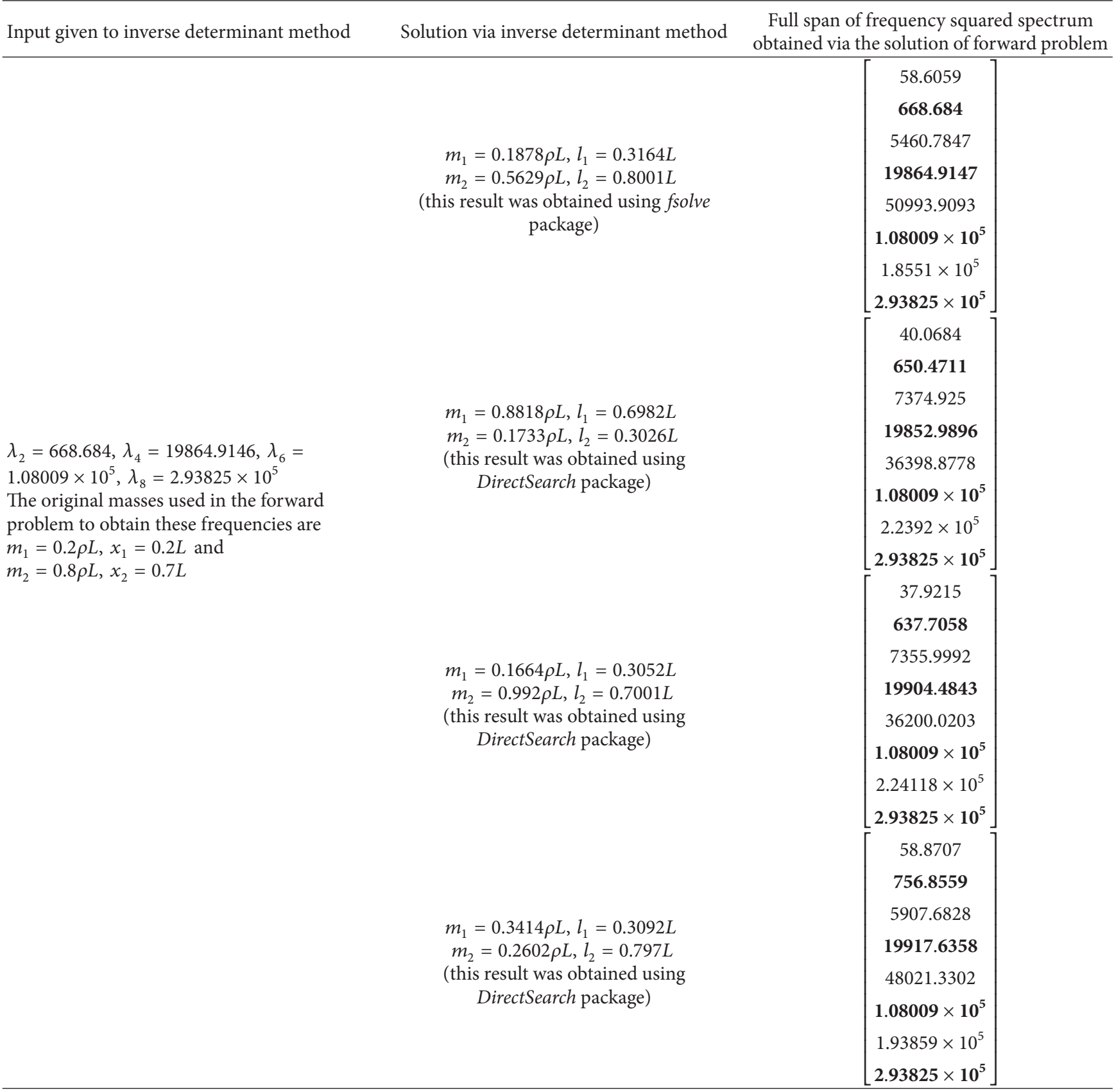

of view of engineering design especially when lower orders of frequencies are involved.

\section{Conflict of Interests}

The authors declare that they have no conflict of interests.

\section{Authors' Contribution}

Farhad Mir Hosseini and Natalie Baddour developed the analytical models in the paper. Farhad Mir Hosseini performed all coding and simulations. Farhad Mir Hosseini and Natalie Baddour drafted the paper.

\section{Acknowledgment}

This work was financially supported by the Natural Science and Engineering Research Council of Canada.

\section{References}

[1] S. Kukla and B. Posiadala, "Free vibrations of beams with elastically mounted masses," Journal of Sound and Vibration, vol. 175, no. 4, pp. 557-564, 1994. 
[2] J. W. Nicholson and L. A. Bergman, "Free vibration of combined dynamical systems," Journal of Engineering Mechanics, vol. 112, no. 1, pp. 1-13, 1986.

[3] E. H. Dowell, "On some general properties of combined dynamical systems," Journal of Applied Mechanics, vol. 46, no. 1, pp. 206-209, 1979.

[4] E. H. Dowell, "Free vibrations of an arbitrary structure in terms of component modes," Journal of Applied Mechanics, vol. 39, no. 3, pp. 727-732, 1972.

[5] K. H. Low, "On the eigenfrequencies for mass loaded beams under classical boundary conditions," Journal of Sound and Vibration, vol. 215, no. 2, pp. 381-389, 1998.

[6] K. H. Low, "Comparisons of experimental and numerical frequencies for classical beams carrying a mass in-span," International Journal of Mechanical Sciences, vol. 41, no. 12, pp. 15151531, 1999.

[7] K. H. Low, "On the methods to derive frequency equations of beams carrying multiple masses," International Journal of Mechanical Sciences, vol. 43, no. 3, pp. 871-881, 2001.

[8] K. H. Low, "Frequencies of beams carrying multiple masses: rayleigh estimation versus eigenanalysis solutions," Journal of Sound and Vibration, vol. 268, no. 4, pp. 843-853, 2003.

[9] P. D. Cha, "A general approach to formulating the frequency equation for a beam carrying miscellaneous attachments," Journal of Sound and Vibration, vol. 286, no. 4-5, pp. 921-939, 2005.

[10] P. D. Cha and N. C. Yoder, "Applying Sherman-MorrisonWoodbury formulas to analyze the free and forced responses of a linear structure carrying lumped elements," Journal of Vibration and Acoustics, vol. 129, no. 3, pp. 307-316, 2007.

[11] D. Wang, "Vibration and sensitivity analysis of a beam with a lumped mass of translational and rotary inertias," Journal of Vibration and Acoustics, vol. 134, no. 3, Article ID 034502, 2012.

[12] J. I. Pritchard, H. M. Adelman, and R. T. Haftka, "Sensitivity analysis and optimization of nodal point placement for vibration reduction," Journal of Sound and Vibration, vol. 119, no. 2, pp. 277-289, 1987.

[13] P. D. Cha and C. Pierre, "Frequency analysis of a linear elastic structure carrying a chain of oscillators," Journal of Engineering Mechanics, vol. 125, no. 5, pp. 587-591, 1999.

[14] P. D. Cha and C. Pierre, "Imposing nodes to the normal modes of a linear elastic structure," Journal of Sound and Vibration, vol. 219, no. 4, pp. 669-687, 1999.

[15] L. Meirovitch, Principles and Techniques of Vibrations, Prentice Hall, Upper Saddle River, NJ, USA, 1997.

[16] P. D. Cha, "A general approach to formulating the frequency equation for a beam carrying miscellaneous attachments," Journal of Sound and Vibration, vol. 286, no. 4-5, pp. 921-939, 2005.

[17] P. Dumond and N. Baddour, "A structured approach to using a rectangular brace to design a aoundboard section for a desired natural frequency," in Proceedings of the Stockholm Music Acoustics Conference, pp. 613-618, Stockholm, Sweden, 2013.

[18] S. Moiseev, "Universal derivative-free optimization method with quadratic convergence," http://arxiv.org/abs/1102.1347.

[19] P. B. Thanedar, J. S. Arora, G. Y. Li, and T. C. Lin, "Robustness, generality and efficiency of optimization algorithms for practical applications," Structural Optimization, vol. 2, no. 4, pp. 203212, 1990.
[20] P. W. Bo and D. Costin, "Optimum design of a composite structure with three types of manufacturing constraints," AIAA journal, vol. 30, no. 6, pp. 1667-1669, 1992.

[21] J. Sobieszczanski-Sobieski and J. Tulinius, "MDO can help resolve the designer's dilemma," Aerospace America, vol. 29, no. 9, pp. 32-63, 1991.

[22] B. E. Richardson, "Acoustical design criteria for the guitar," Journal of the Acoustical Society of America, vol. 97, no. 5, article 3354, 1995.

[23] J. Natelson and W. Cumpiano, Guitarmaking: Tradition and Technology: A Complete Reference for the Design \& Construction of the Steel-String Folk Guitar \& the Classical Guitar, Chronicle Books, San Francisco, Calif, USA, 1994.

[24] P. Dumond and N. Baddour, "Can a brace be used to control the frequencies of a plate?" Springer Plus, vol. 2, no. 1, article 558, 2013. 


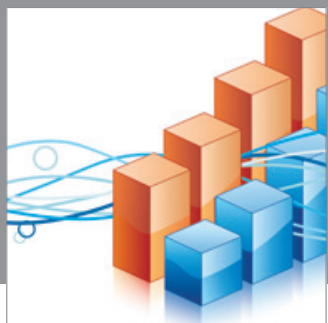

Advances in

Operations Research

mansans

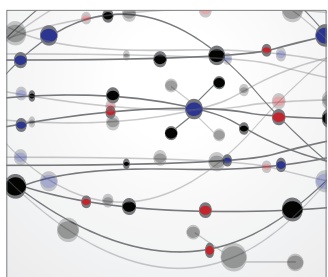

The Scientific World Journal
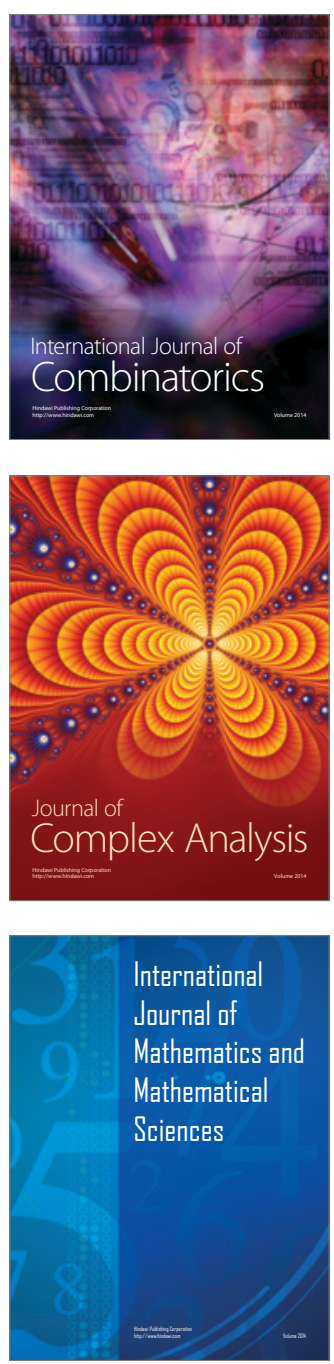
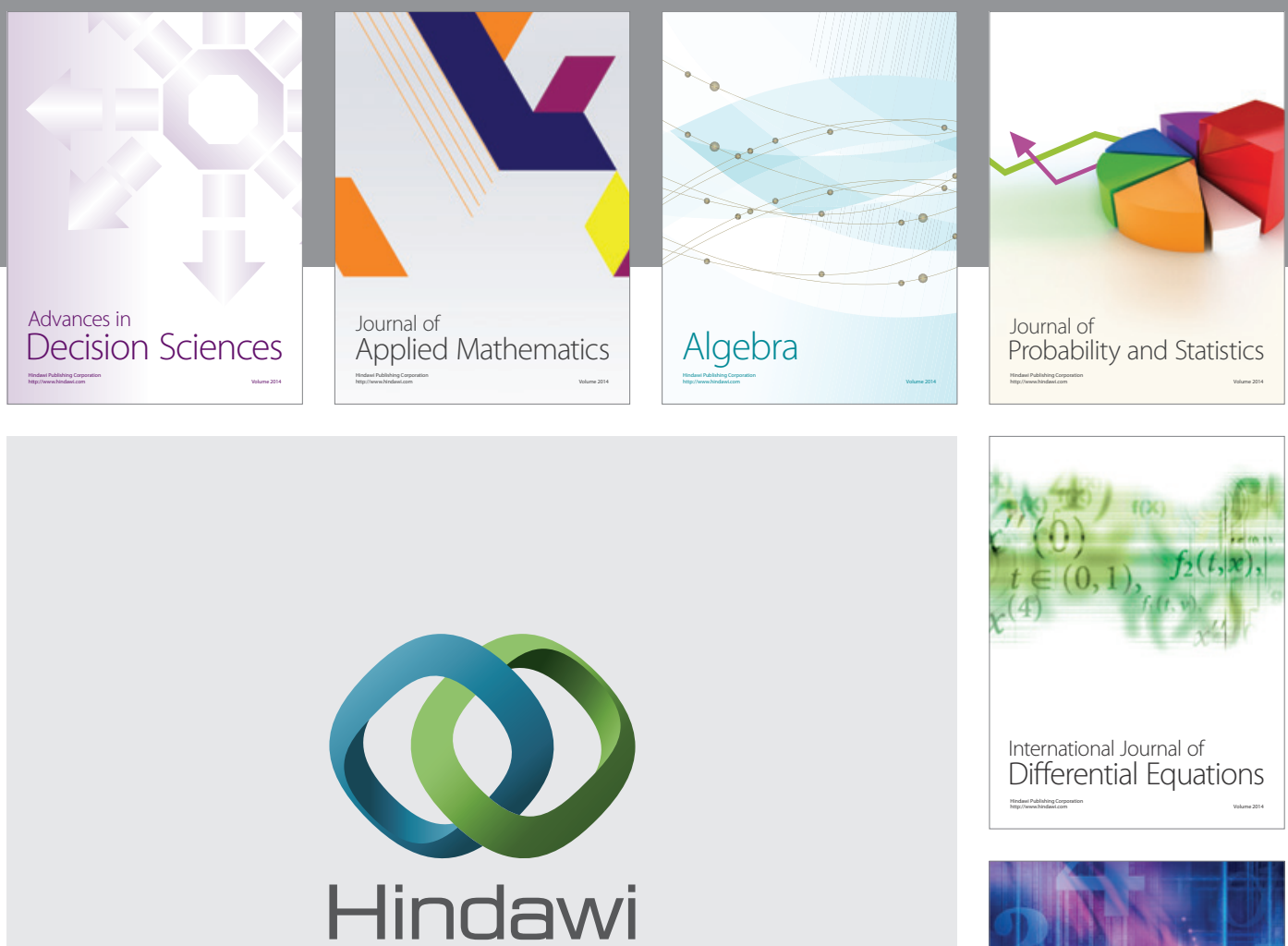

Submit your manuscripts at http://www.hindawi.com
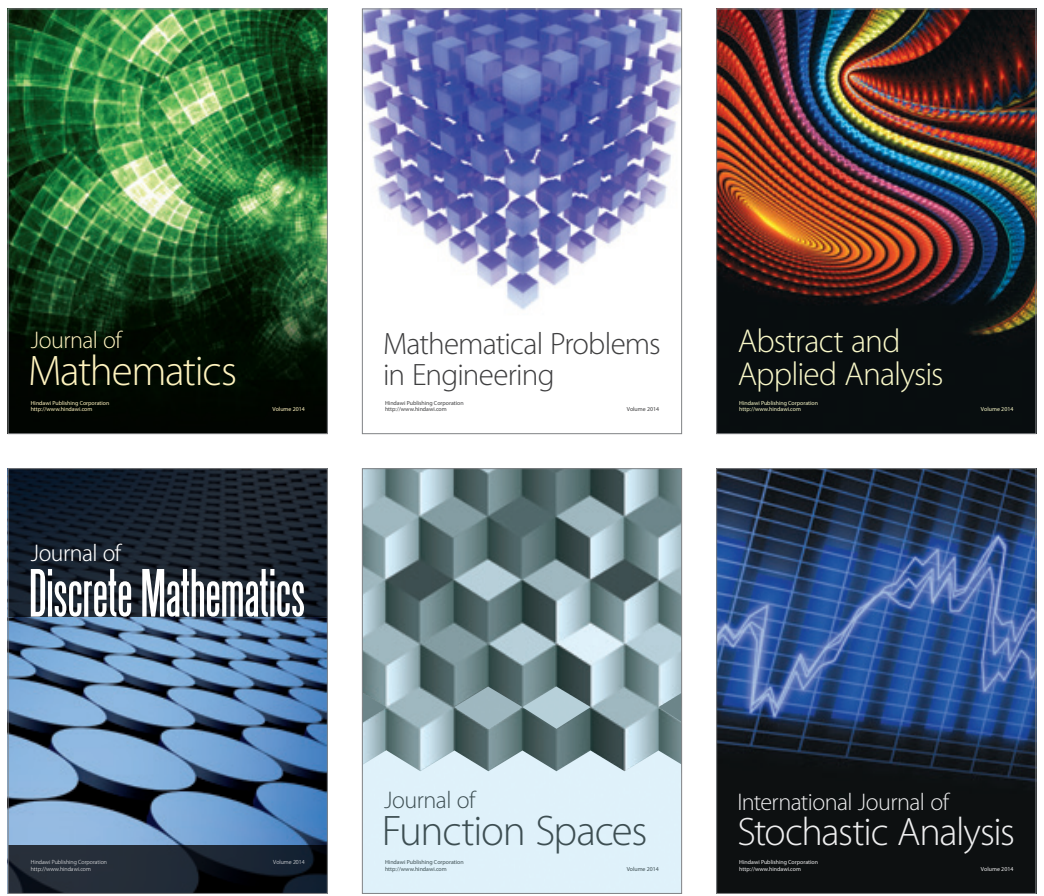

Journal of

Function Spaces

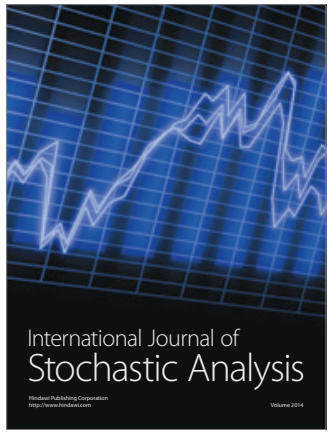

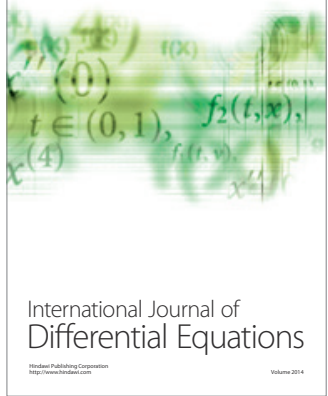
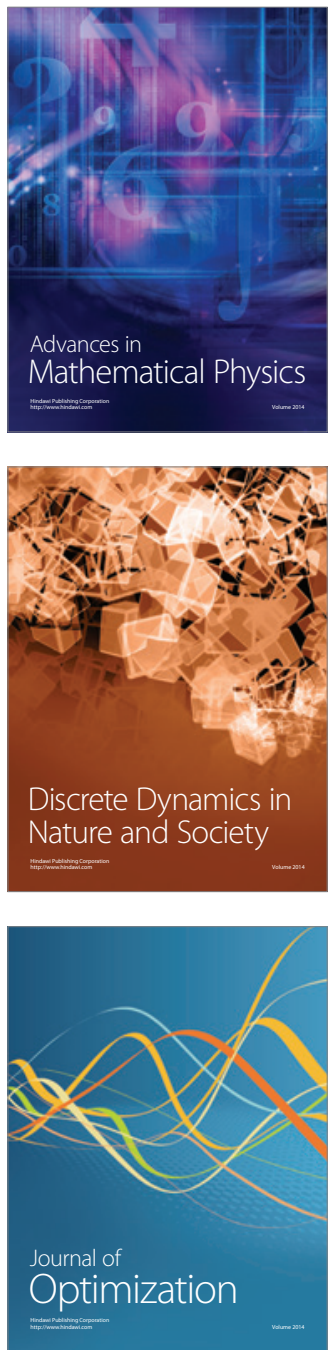